\title{
FUNCTION OF A PERIODIC VARIABLE GIVEN BY THE STEADY READING OF AN INSTRUMENT; WITH A NOTE ON THE USE OF THE CAPILLARY ELECTROM- ETER WITH ALTERNATING VOLTAGES.
}

\author{
By Morton G. Lloyd.
}

This investigation was made in the hope of finding an instrument which would register the average (numerical) value of an alternating electromotive force, but an examination of the conditions shows that no instrument can answer this purpose unless its deflection is independent of the direction of the applied voltage and proportional to that voltage in numerical magnitude.

In instruments whose parts have relative motion, as a galvanometer, the force exerted depends upon the position of the moving part and upon the variable being measured, which for brevity will be referred to as the voltage. In others, such as the Siemens dynamometer or the capillary electrometer with uniform bore (or in which the hydrostatic pressure is varied to keep the meniscus stationary), the force depends upon the voltage alone.

In either case, if the moving part maintains a fixed position during the variations of the voltage, the variations of force during a period depend only upon the variations of the voltage, and the average value of that force is what determines the deflection and is measured by it. The function given by the instrument reading consequently depends only upon the law of force involved. Let us suppose that the force is such a function of the voltage that it may be expressed by a series of powers.

$$
F=A_{\mathrm{o}}+A_{1} e+A_{2} e^{2}+A_{3} e^{3}+\text {. . . . . . . }
$$


The average value of the force during a period, $T$, is

$$
\frac{I}{T} \int_{t}^{t+T} F d t=A_{0}+\frac{A_{1}}{T} \int_{t}^{t+T} e d t+\frac{A_{2}}{T} \int_{t}^{t+T} e^{2} d t+\ldots . . .
$$

The terms involving odd powers of $e$ disappear, for

$$
\int_{t}^{t+\frac{T}{2}} e^{\mathrm{m}} d t=-\int_{t+\frac{T}{2}}^{t+T} e^{m} d t \text { and hence } \int_{t}^{t+T} e^{m} d t=0
$$

when $m$ is an odd integer.

Since $A_{\mathrm{o}}$ is the force corresponding to the zero reading, we have for the force due to the voltage

$$
F-A_{\mathrm{o}}=\frac{A_{2}}{T} \int_{t}^{t+T} e^{2} d t+\frac{A_{4}}{T} \int_{t}^{t+T} e^{t} d t+\ldots . . .
$$

and it is evidently impossible that the instrument should indicate the average value of the voltage.

Let us consider the relation between deflection and voltage to be parabolic, as is approximately the case with the capillary electrometer, so that

$$
D=b-\frac{b}{a^{2}}(e-a)^{2}=2 \frac{b}{a} e-\frac{b}{a^{2}} e^{2}
$$

where $b$ is the maximum positive value of $D$, occurring when $e=a$. (See Fig. I.) Then since the law of force is here the same as the

where

$$
F-A_{\mathrm{o}}=-\frac{I}{T} \int_{t}^{t+T} \frac{b}{a^{2}} e^{2} d t=-\frac{b}{a^{2}} E^{2}
$$

$$
E=\sqrt{\frac{r}{T} \int_{t}^{t+T} e^{2} d t}
$$

law of deflection and may be designated the effective value of $e$. 
The deflection in this case is always negative and follows a parabolic law of variation with $E$, in approximate agreement with the experimental results (see p. 529).

An instrument can only give readings proportional to the numerical average value of $e$ by having the coefficient $A_{1}$ change sign with $e$, and $A_{2}=O=A_{4}=$ etc. This can be accomplished in two ways; first, by reversal of the force at the instant $e$ is passing through the valne zero; secondly, by making the permanent factor in the deflecting force controlled as to direction by the variable factor. The first

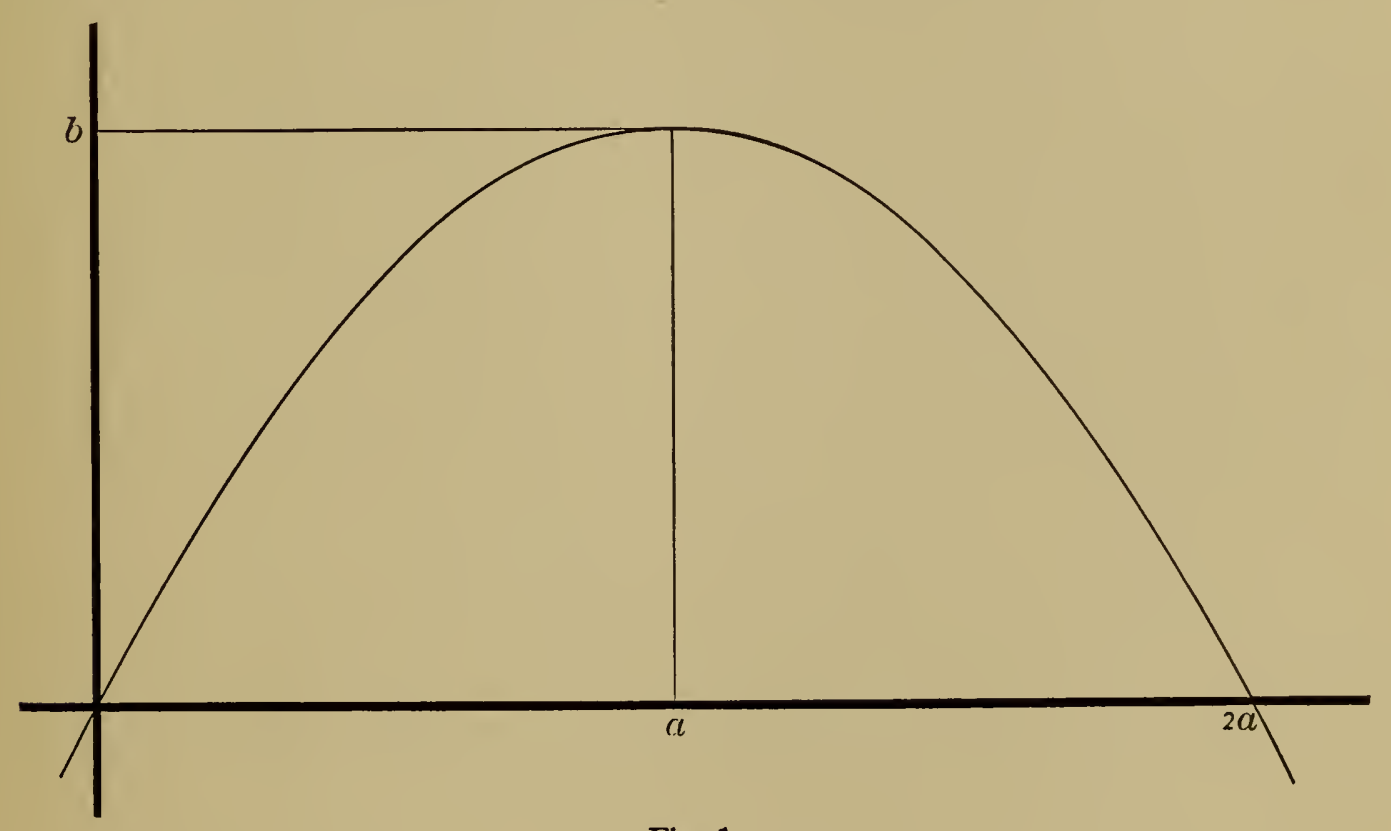

Fig. 1.

method has been applied in an apparatus already described in this Bulletin. ${ }^{1}$ The second method is difficult of application, but a hypothetical case may be mentioned by way of illustration.

Let the moving element in the instrument be a soft iron needle surrounded by a coil of wire of sufficient turns, so that the smallest current to be measured-in fact, a small percentage of its maximum value-will magnetize it to saturation. Let the current to be measured traverse also a coil so placed that its field will deflect the magnetized needle. Then the torque acting on the needle will be constant in direction and proportional to the current. Besides

${ }^{1}$ Lloyd and Fisher, Bull. 4, p. 467; 1908, where a rotating commutator in synchronism with an A. C. generator is used.

22832-08-5 
being inpracticable on account of the prohibitive inductance necessary, this scheme is open to certain theoretical objections, but it will serve as an illustration of the necessities of the case.

Dynamometer ammeters for alternating currents have been devised $^{2}$ in which the deflection is proportional to the current, since by the positions of the coils the force is made to vary inversely as the deflection.

It n1ay seem at first sight as though the indication of such an instrument would depend upon the average value of the current, but it has been shown above that it would depend upon the effective value. The reason for this is, that it is the force which is integrated, and the deflection is determined by the average value of the force, and not by the average value of the deflections which would result if the variable were to proceed very slowly through a cycle.

If $D$ be the deflection, $i$ the instantaneous current, $F=k_{1} \frac{i^{2}}{D}$, and since the controlling force is proportional to the deflection, we have

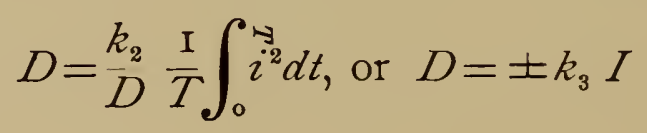

where $I$ is the effective value of the current.

${ }^{2}$ H. Bruger, Phys. Zs., 4, p. 876 ; 1903. 


\section{ON THE USE OF THE CAPILLARY ELECTROMETER WITH ALTERNATING VOLTAGES.}

The capillary electrometer, introduced by Lippmann ${ }^{3}$ in 1873 , has been used extensively by physiologists and physical chemists, ${ }^{*}$ and more lately has received attention as a laboratory instrument for more general use. ${ }^{5}$

This form of electrometer has been used more generally as a null instrument, but can also be used for deflections, if the scale be calibrated. Calibration is necessary, since except for very small ranges the deflection is not proportional to the applied voltage. When used as a deflecting instrument, the mercury in the capillary is usually charged negatively and the electrolyte positively, but this is not necessary, as deflections can be obtained in either direction.

So far as is known to the author, the instrument has never heretofore been used with alternating potentials, except in the wavetracing experiments of Burch. His instrument was designed to have short period and to be dead beat, and very low voltages were used.

If a curve be plotted between deflections and applied direct voltages, it has the general form of a parabola, as shown in Fig. 3.

It was surmised from this that the instrument would respond to an alternating voltage with a deflection, and such was found to be the case.

The electrometer was set up, as shown in Fig. 2, the beaker having a layer of mercury at the bottom covered with the electrolyte. A glass tube was drawn to a capillary and bent as shown, then partly filled with mercury and the capillary end immersed in the electrolyte. Platinum wires dipping into the mercury formed the electrodes, and were protected from contact with the electrolyte. A inicrometer microscope was focussed upon the surface separating

\footnotetext{
${ }^{3}$ G. Lippmann, Pogg. An11., 149, p. 546; 1873 .

${ }^{4} \mathrm{~W}$. Ostwald, Zs. f. Phys. Chem., 1, p. 404; 1887, and Ostwald and Luther's Physiko-chemische Messungen, 2te Auflage, p. 333.

${ }^{5} \mathrm{G}$. J. Burch, London Electrician, 37, p. 380 ; 1896.

C. F. Burgess, Trans. A. I. E. E., 15, p. 337; 1898.

A. D. Cole, Bull. Scientific Lab. Denison Univ., 11, p. 265; 1902.

A. W. Vining, Ann. chim. phys., 9, p. 272; 1906.
} 
the two liquids in the capillary, and any change in the position of the meniscus due to electrification could be measured.

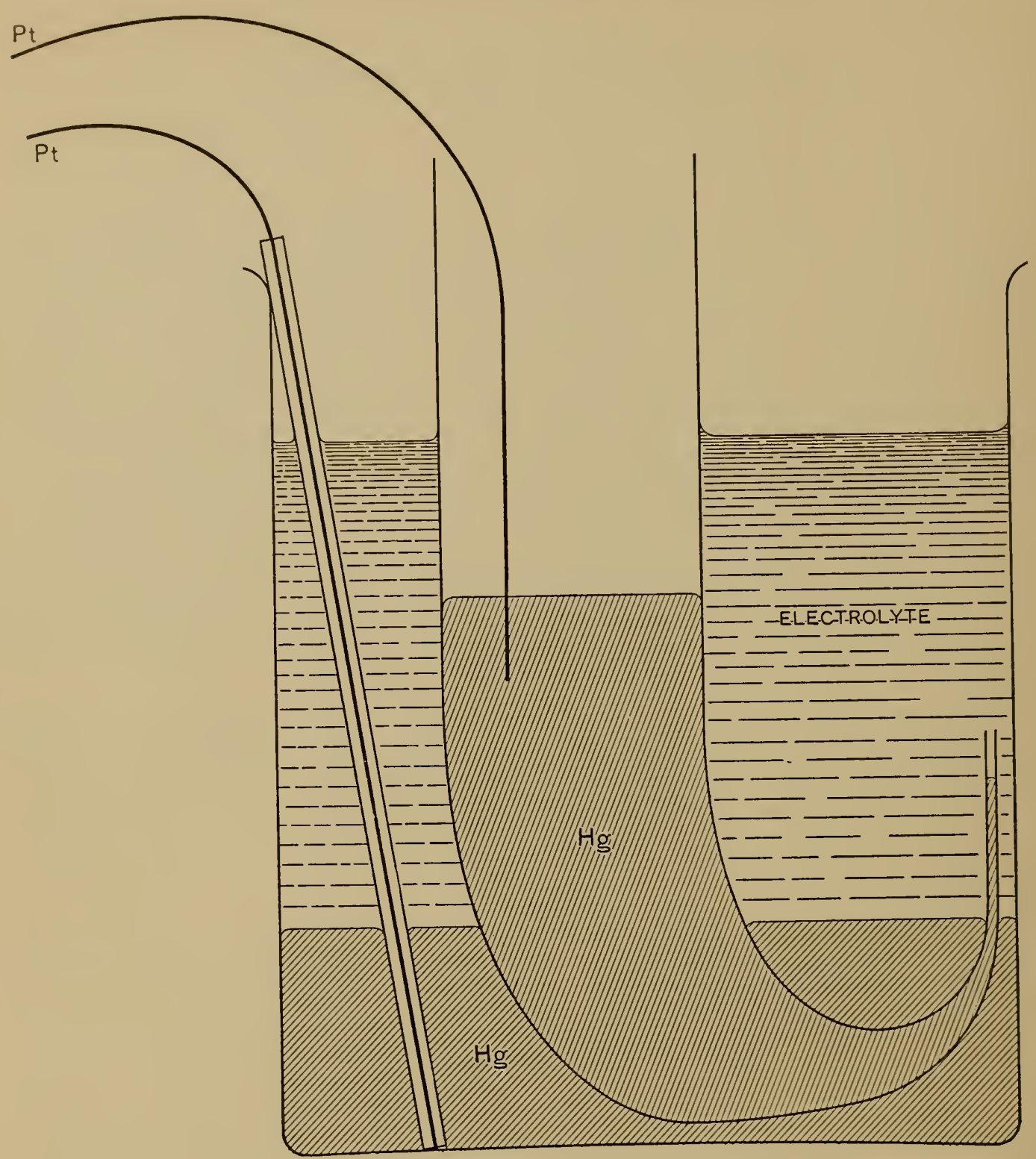

Fig. 2.

The electrolyte used was an aqueous solution of sodium chloride. This was chosen as less likely to gas at low voltages than sulphuric acid, the electrolyte usually employed. ${ }^{6}$ The diameter of bore at the surface of separation was about 0.7 millimeter. A finer bore was avoided in order to lessen trouble in case of gassing.

${ }^{6}$ F. Paschen, Wied. Ann., 39, p. 43 ; I89o. 


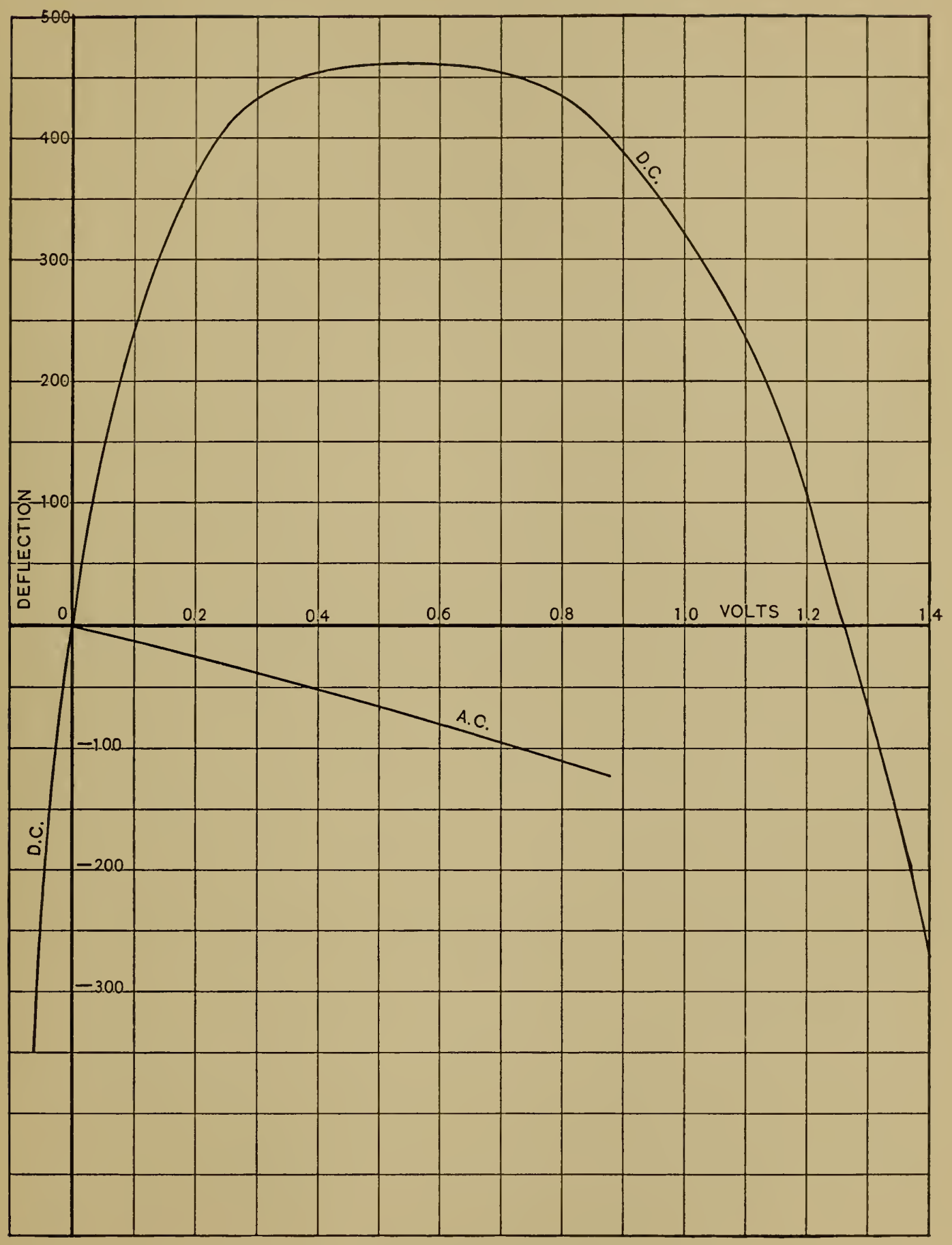

Fig. 3. 
The electrometer was first calibrated with direct voltage, and the results are shown graphically in Fig. 3, curve D. C. Alternating voltages were next applied, with the results shown in curve A. C., whose abscissæe are effective volts, as read upon a dynamometer. Direct voltages are considered positive when the mercury in the capillary is connected to the negative pole of the battery. With direct voltages the position of the meniscus was perfectly definite and reproducible. With alternating voltages the readings, except for very small voltage, are not steady and are not reproducible. The set given in the figure is simply a sample of their general value. On acconnt of this uncertainty in deflection the electrometer could not be used for an accurate measurement of alternating voltage. This difficulty is probably due to the fact that the surface never comes to rest, but a photographic record would be necessary to detect the motion.

As a detector of alternating voltages, it would be perfectly feasible to use the electrometer. The sensibility, however, is very much smaller for A. C. than for D. C. on the same instrument, and it does not maintain its advantages in this field of work. In an age when a telephone is a universal laboratory instrument, the electrometer can hardly be expected to attain very general use. There are two features of this instrument, however, which should be kept in mind with regard to its use for both direct and alternating voltages. These are the fact that it is an electrostatic instrument, requiring only sufficient current to charge or polarize it, and its cheapness. In many laboratories where the cost of more elaborate apparatus is prohibitive, the capillary electrometer may be made to answer many purposes.

Washington, December 30, I907. 